\title{
VISIBLE WATERMARKING WITHIN THE REGION OF NON-INTEREST OF MEDICAL IMAGES BASED ON FUZZY C-MEANS AND HARRIS CORNER DETECTION
}

\author{
Debalina Biswas ${ }^{1}$, Poulami Das ${ }^{2}$, , Prosenjit Maji ${ }^{3}$, \\ Nilanjan Dey ${ }^{4}$, Sheli Sinha Chaudhuri ${ }^{5}$ \\ ${ }^{1,2,3}$ Department of CSE, JIS College of Engineering, Kalyani, West Bengal, \\ India \\ poulamidas1989@gmail.com,biswas_debalina@rediffmail.com, \\ maji_katmerediffmail.com \\ ${ }^{4}$ Department of IT, JIS College of Engineering, Kalyani, West Bengal, India \\ dey.nilanjan@ymail.com \\ ${ }^{5}$ ETCE Department, Jadavpur University, Kolkata, India \\ shelismerediffmail.com
}

\begin{abstract}
Transfer of medical information amongst various hospitals and diagnostic centers for mutual availability of diagnostic and therapeutic case studies is a very common process. Watermarking is adding "ownership" information in multimedia contents to verify signal integrity, prove authenticity and achieve control over the copy process. Distortion in Region of Interest (ROI) of a bio-medical image caused by watermarking may lead to wrong diagnosis and treatment. Therefore, proper selection of Region of Non-Interest (RONI) in a medical image is very crucial for adding watermark. First part of the present work proposes proper selection of Region of Non-Interest based on Fuzzy C-Means segmentation and Harris corner detection, to improve retention of diagnostic value lost in embedding ownership information. The second part of the work presents watermark embedding in the selected area of RONI based on alpha blending technique. In this approach, the generated watermarked image having an acceptable level of imperceptibility and distortion is compared to the original image. The Peak Signal to Noise Ratio (PSNR) of the original image vs. watermarked image is calculated to prove the efficacy of the proposed method.
\end{abstract}

\section{KEYWORDS}

Fuzzy C-Means, Harris Corner Detection, ROI, RONI

\section{INTRODUCTION}

In telemedicine, medical images i.e. electro digital data are continuously sent to and received at various health care centres and hospitals around the world. The obvious constraint faced in this situation is to detect the organization from which the particular image has been sent and / or received. Solution to this problem is watermarking $[1,2,3,4]$ the name of the medical centre on the digitized image of the referred patient. Apart from this, EPR (Electronic Patient Report) watermarking $[5,6,7]$ is also a common form of medical data exchange with authenticity in

David C. Wyld (Eds) : ICCSEA, SPPR, CSIA, WimoA - 2013

pp. 161-168, 2013. (C CS \& IT-CSCP 2013

DOI : $10.5121 /$ csit.2013.3517 
telemedicine. However, medical watermarking $[8,9,10,11]$ has its own limitations and requires a sophisticated execution such that minimal data is tampered.

The most informative area of the medical image is called as Region of Interest (ROI). ROI [12, 13] is the area used for diagnosis by the physician. Any distortion in ROI may lead to wrong diagnosis and treatment. Hence, for watermarking medical images, area outside ROI in the image should be chosen. This part of the image is called as Region of Non Interest (RONI).

With the onset of modern medical science, use of several imaging techniques has become the basement for diagnosis of diseases among the medical professionals. Some of the imaging techniques are, Magnetic Resonance Imaging (MRI), Computed Tomography (CT), Ultrasound (US), Skiagram, Positron Emission Tomography (PET), Mammogram, Intravascular Ultrasound (IVUS) etc.

Intravascular ultrasound is a medical imaging technique, which uses an ultrasound probe positioned at the tip of a coronary catheter. Intravascular ultrasound is a tomographic imaging modality performed during coronary angiography. In case of IVUS, ultrasound signal reflected from the surrounding tissue is reconstructed into a real-time tomographic gray scale image [14]. IVUS can identify the boundaries of different layers of blood vessels such as internal elastic intima and external elastic intima. Borders between tissues with different composition and acoustic properties cause strong ultrasound reflections. Two such borders in the coronary artery walls are the lumen/intimal border and external elastic membrane. The strong signal originating at these tissue interfaces allows recognition and manual or automated planimetry. Thus, IVUS becomes capable of measuring lumen area by identified borders of lumen and intima. By measuring maximum and minimum diameter of External Elastic Lamina, IVUS makes it possible to diagnose atherosclerotic arteries. IVUS can calculate atheroma through EEM and Lumen CSA measurements i.e. plaque plus media area [15]. Comprehensively, it can be said that IVUS is capable of measuring maximum and minimum thickness of plaque along with media, plaque plus media eccentricity and plaque burden. Length of plaque can be measured by the motorized transducer pullback function.

MRI (Magnetic Resonance Imaging) is a noninvasive medical technique, which helps the physicians to diagnose the patients, detect abnormalities and treat medical conditions. It is used to detect structural abnormalities of the body. MRI is capable of giving images of different organs and structures inside the body in more detail than X-Ray, ultrasound and CT- Scan. MRI is a radiology technique that uses magnetism, radio waves and a computer for imaging different parts of the body $[16,17]$. MRI of different parts of the body is suggested for different reasons. MRI of head is suggested for detecting brain tumors, injuries in the brain, nerve injury, bleeding in the brain. Spine MRI can check the discs and nerves of the spine for conditions such as spinal stenosis, disc bulges, and spinal tumors. Chest MRI is done to examine the heart, valves and lungs. Chest MRI can detect abnormalities in heart and lungs functioning, lung cancer and breast cancer too. MRI is also capable of imaging blood vessels to detect any abnormalities in the flow of blood through the vessels. Gall bladder, kidneys, liver, pancreas can also be examined by MRI for detecting abnormalities [18]. MRI is often suggested for fetal assessment in pregnant women. While doing MRI the patient is placed on a moveable bed. Then the bed is inserted into a magnet, which creates a strong magnetic field. The magnetic field aligns the protons of hydrogen atoms, which are then exposed to a beam of radio waves. This spins the various protons of the body, and they produce a faint signal that is detected by the receiver portion of the MRI scanner. The receiver information is processed by a computer, and an image is produced. The image and resolution produced by MRI is quite detailed and can detect tiny changes of structures within the body. For some procedures, contrast agents, such as gadolinium, are used to increase the accuracy of the images. 
ROI and RONI marked IVUS and MRI image is shown in the figure 1.

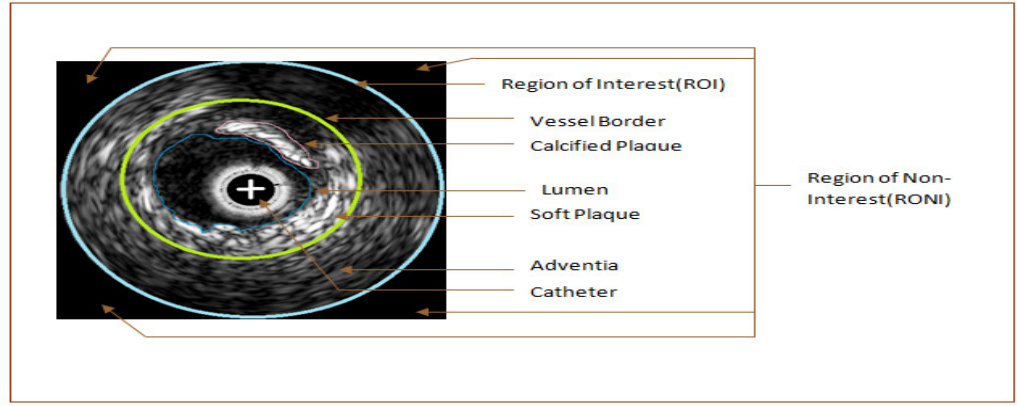

(a)

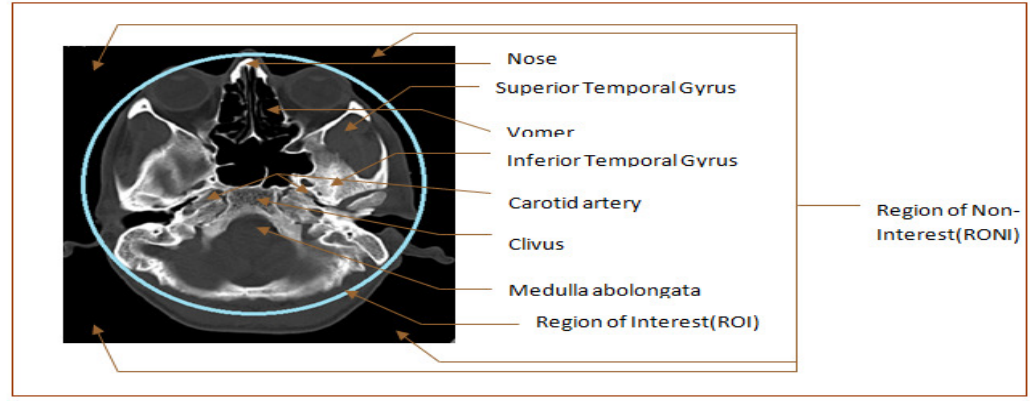

(b)

Figure 1. (a) ROI and RONI marked IVUS image, (b) ROI and RONI marked MRI image

\section{Methodology}

\subsection{Fuzzy C-Means}

In pattern recognition, a clustering method known as Fuzzy c-means (FCM) is widely used. Bezdek proposed FCM in 1973[19, 20]. FCM is also known as Fuzzy ISODATA. In this clustering technique, one piece of data belongs to two or more clusters. FCM based segmentation is fuzzy pixel classification. FCM allows data points or pixels to belong to multiple classes with varying degree of membership function between 0 and 1 .

FCM possesses unique advantage of grading linguistic variables to fit for appropriate analysis in discrete domain on pro-rata basis.

FCM computes cluster centers or Centroids by minimizing the dissimilarity function with the help of iterative approach. By updating the cluster centers and the membership grades for individual pixel, FCM [21] shifts the cluster centers to the "right" location within set of pixels.

To accommodate the introduction of fuzzy partitioning, the membership matrix $(U)=\left[\mathrm{u}_{\mathrm{ij}}\right]$ is randomly initialized according to Equation 1, where $\mathrm{u}_{\mathrm{ij}}$ being the degree of membership function of the data point of $i^{\text {th }}$ cluster $\mathrm{x}_{\mathrm{i}}$.

$$
\sum_{i=1}^{c} u_{i j}=1, \forall j=1, \ldots, n
$$

The performance index (PI) for membership matrix U and ci's used in FCM is given Equation 2. 


$$
J\left(U, c_{1}, c_{2}, \ldots, c_{c}\right)=\sum_{i=1}^{c} J_{i}=\sum_{i=1}^{c} \sum_{j=1}^{n} u_{i j}{ }^{m} d_{i j}{ }^{2}
$$

$\mathrm{u}_{\mathrm{ij}}$ is between 0 and 1 .

$c_{i}$ is the centroid of cluster $i$.

$\mathrm{d}_{\mathrm{ij}}$ is the Euclidian distance between $\mathrm{i}^{\text {th }}$ centroid (ci) and $\mathrm{j}^{\text {th }}$ data point.

$m \in[1, \infty]$ is a weighting exponent.

To reach a minimum of dissimilarity function there are two conditions. These are given in Equation 3 and Equation 4.

$$
\begin{gathered}
c_{i}=\frac{\sum_{j=1}^{n} u_{i j}{ }^{m} x_{j}}{\sum_{j=1}^{n} u_{i j}{ }^{m}} \\
u_{i j}=\frac{1}{\sum_{k=1}^{c}\left(\frac{d_{i j}}{d_{k j}}\right)^{2 /(m-1)}}
\end{gathered}
$$

\subsubsection{Algorithm of FCM}

Step 1. The membership matrix (U) that has constraints in Equation 1 is randomly initiated.

Step 2. $\quad$ Centroids (ci) are calculated by using Equation 3

Step 3. Dissimilarity between centroids and data points is computed using Equation 2. Stop if its improvement over previous iteration is below a threshold.

Step 4. $\quad$ A new U is computed using Equation 4. Go to Step 2.

\subsection{Alpha Blending Technique}

Alpha Blending $[22,23]$ is a well known technique in computer graphics for blending each pixel of the first source image with the corresponding pixel in the second source image. The equation for executing alpha blending is as follows,

Final pixel $=$ alpha $*($ First image's source pixel $)+(1.0$-alpha $) *($ Second image's source pixel $)$ The blending factor or percentage of colors from the first source image used in the blended image is called the "alpha". The alpha used in algebra is in the range 0.0 to 1.0, instead of 0 to $100 \%$. Alpha-blending blind Image hiding technique to generate Watermark image is given by Watermark Embedding:

$$
\text { WII=alpha*(CI) }+(1.0 \text {-alpha }) *(\mathrm{WI})
$$

where, WII=watermarked image, $\mathrm{CI}=$ selected sub-band of the cover image, WI= selected corresponding sub-band of the watermark image. 


\section{Proposed Method}

Watermark embedding process is explained in Figure 2.

Step 1. $\quad$ Fuzzy C-means Segmentation is applied on gray medical image.

Step 2. Segmented Image is binarized.

Step 3. Binary area open is used to remove small objects from binarized image.

Step 4. Sobel Edge detection is applied on the binarized image.

Step 5. Harris Corner Detection Algorithm [24, 25] is applied.

Step 6. Maximum Harris Diameter is calculated and based on the Harris points the centre is determined.

Step 7. A circle is drawn based on the centre point and diameter on the sample image to mark the boundary between Region of Interest (ROI) and Region of Non-Interest (RONI).

Step 8. An area from the RONI is selected based on the watermark image size.

Step 9. Watermark image is embedded on the selected area based on alpha blending technique.

The watermarked image is overlaid on the selected position of the original image to generate the Final watermarked Image.

\section{RESUlt AND DisCUSSION}

MATLAB 7.0.1 Software is extensively used for the study of the medical image watermarking embedding technique. Concerned images obtained in the result are shown in Figure 2 through 6.

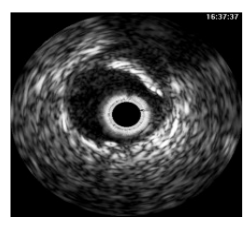

(a)

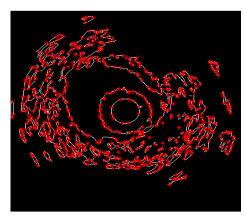

(e)

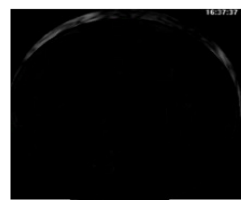

(i)

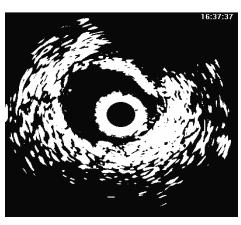

(b)

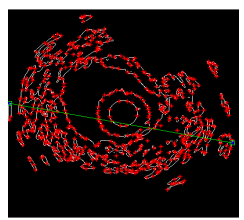

(f)

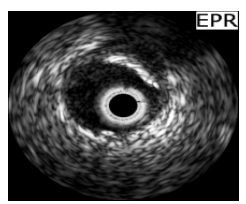

(j)

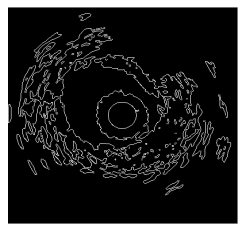

(c)

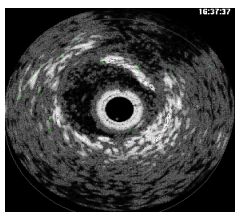

(g)

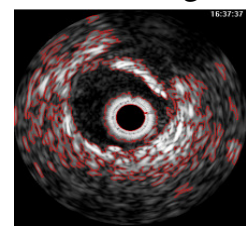

(d)

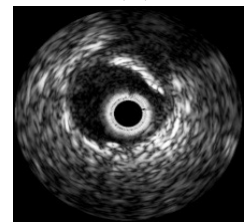

(h)

Figure 2. (a) Original IVUS Image, (b) Fuzzy C-Means Segmented Image, (c) Edge detected Image, (d) Marked ROI, (e) Harris Corner Detected Image, (f) Maximum Harris Diameter, (g) Marked ROI using Harris Corner detection, (h) ROI, (i) RONI, (j) Watermark Embedded Image 


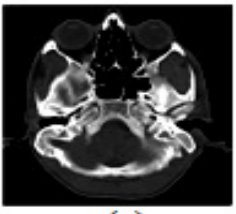

(a)

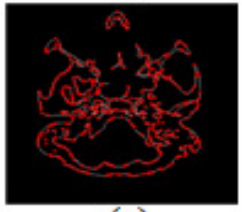

(e)

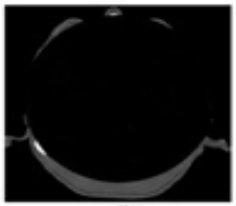

(i)

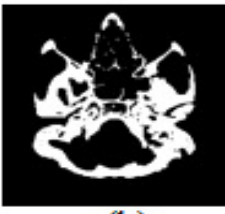

(b)

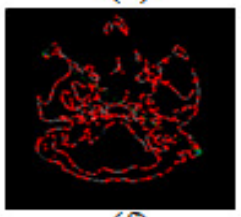

(f)

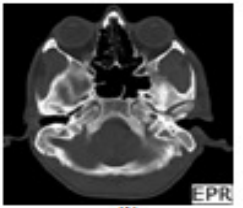

(j)

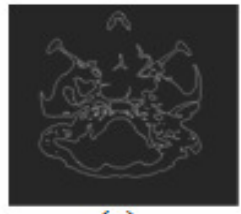

(c)

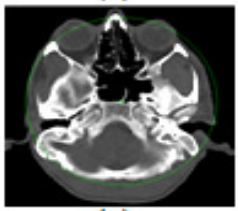

(g)

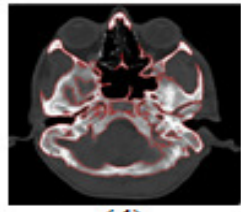

(d)

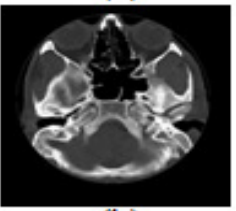

(h)

Figure 3. (a) Original MRI Image, (b) Fuzzy C-Means Segmented Image, (c) Edge detected Image, (d) Marked ROI, (e) Harris Corner Detected Image, (f) Maximum Harris Diameter, (g) Marked ROI using Harris Corner detection, (h) ROI, (i) RONI, (j) Watermark Embedded Image

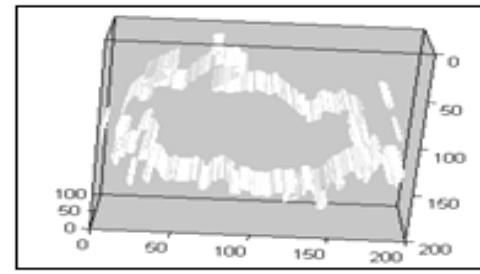

(a)

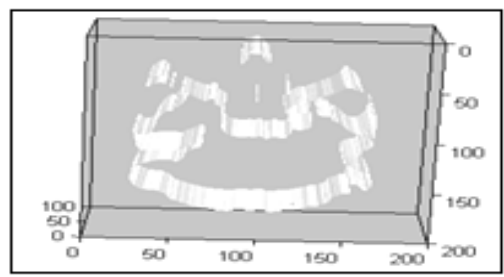

(b)

Figure 4. (a) 3D Reconstruction of the detected boundary between ROI and RONI of IVUS image, (b) 3D Reconstruction of the detected boundary between ROI and RONI of MRI image.

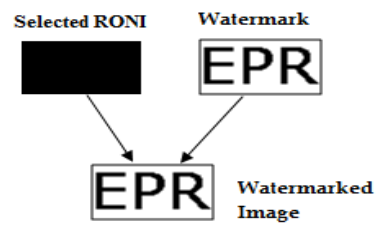

Alpha $=0.99$

Figure 5. Watermark Embedding Using Alpha-Blending Technique

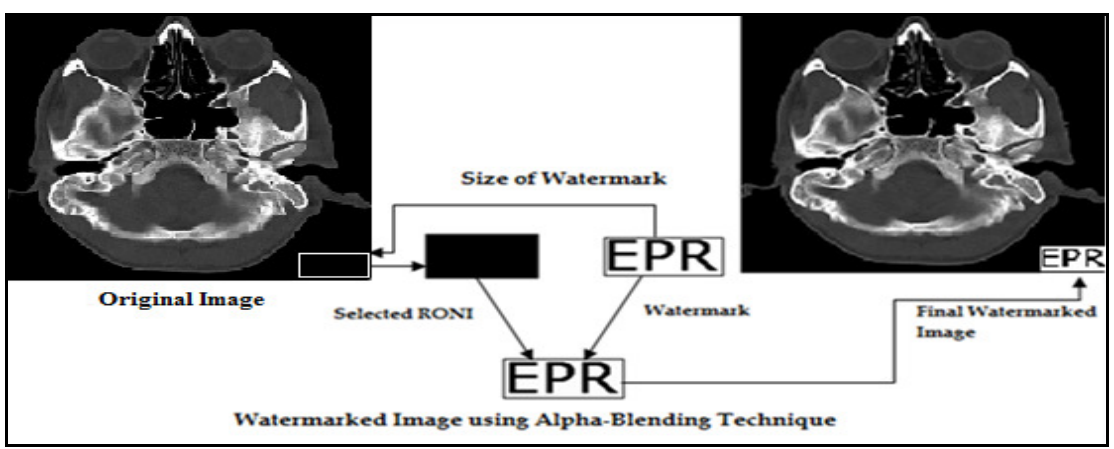

Figure 6. Watermark Embedding into the RONI of Medical Image 


\section{Peak Signal to Noise Ratio (PSNR)}

It measures the quality of a watermarked signal. This performance metric is used to determine perceptual transparency of the watermarked signal with respect to original signal [26]:

$$
P S N R=\frac{M N \max _{x, y} P_{x, y}^{2}}{\sum_{x, y}\left(P_{x, y}-\bar{P}_{x, y}\right)^{2}}
$$

where, $\mathrm{M}$ and $\mathrm{N}$ are number of rows and columns in the input signal,

$$
P_{x, y} \text { is the original signal and } \bar{P}_{x, y} \text { is the watermarked signal. }
$$

PSNR between the Original MRI Image Watermark MRI Image is 28.8255 and PSNR between the Original IVUS Image Watermark IVUS Image is 23.5042 as shown in Table 1.

Table 1.

\begin{tabular}{|l|l|}
\hline PSNR \\
\hline Original MRI Image Vs. Watermark MRI Image & 28.8255 \\
\hline Original IVUS Image Vs. Watermark IVUS Image & 23.5042 \\
\hline
\end{tabular}

\section{CONCLUSION}

It is quite common to exchange medical information between various hospitals and diagnostic centres for mutual availability of diagnostic and therapeutic case studies. EPR (Electronic patient record) or Hospital Logo can be hidden within a bio medical image for high security than transferring EPR/Logo through the internet. As the bio medical images like MRI, CT Scan, USG images convey information for detecting and diagnosis of diseases, exchanging patients' records hidden within these images of patients' diagnostic results requires high reliability. That is why it is necessary to find out such regions of these images, which do not contain diagnostic information. In this paper, region of non-interest (RONI) is found out by using Fuzzy C-means algorithm, which is a clustering method. After identifying the RONI, data is hidden within that region using watermark embedding. As data is hidden within less informative region, distortion of that region of the image due to watermark embedding does not cause any loss in the diagnostic value of the medical image.

\section{REFERENCES}

[1] Jessica Fridrich, Miroslav Goljan, Rui Du,'Invertible authentication" In Proc. SPIE, Security and Watermarking of Multimedia Contents III, Vol. 3971, pp. 197-208, San Jose, USA. (Jan. 2001).

[2] Gouenou Coatrieux, Main, H., Sankur, B., Rolland, Y., \& Collorec. R.'Relevance of watermarking in medical imaging" In IEEE-embs Information Technology Applications in Biomedicine, pp. 250-255, Arlington, USA. (Nov. 2000).

[3] Nilanjan Dey, Moumita Pal,Achintya Das,"A Session Based Watermarking technique Within the NROI of Retinal Fundus Images for Authencation Using DWT,Spread Spectrum and Harris Corner Detection", International Journal of Modern Engineering Research, Vol.2, Issue 3, May-June 2012, pp-749-757 ( ISSN: 2249-6645) .

[4] Nilanjan Dey, Poulami Das, Achintya Das, Sheli Sinha Chaudhuri, "Feature Analysis for the BlindWatermarked Electroencephalogram Signal in Wireless Telemonitoring Using Alattar's Method", 5th International Conference on Security of Information and Networks (SIN 2012), In Technical Cooperation 
with ACM Special Interest Group on Security, Audit and Control (SIGSAC). Proceedings by ACM Press and Digital Library, 22-26 October 2012, Jaipur, India.

[5] Nilanjan Dey, Sayantan Mukhopadhyay, Achintya Das, Sheli Sinha Chaudhuri "Analysis of P-QRS-T Components Modified by Blind Watermarking Technique Within the Electrocardiogram Signal for Authentication in Wireless Telecardiology Using DWT", International Journal of Image, Graphics and Signal Processing, vol. 4,2012.

[6] Nilanjan Dey, Anamitra Bardhan Roy, Achintya Das, Sheli Sinha Chaudhuri," Stationary Wavelet Transformation Based Self-Recovery of Blind-Watermark from Electrocardiogram Signal in the Wireless Telecardiology", International Workshop on Intelligence and Security Informatics for International Security (IIS'12) , 11-12 October 2012, Trivandrum, India, [ Springer in Communications in Computer and Information Science Series(CCIS), ISSN: 1865:0929. CCIS is abstracted/indexed in ISI Proceedings, Scopus, EI and DBLP ].

[7] Nilanjan Dey, Prosenjit Maji , Poulami Das, Achintya Das, Sheli Sinha Chaudhuri, "An Edge Based Watermarking Technique of Medical Images without Devalorizing Diagnostic Parameters”, International Conference on Advances in technology and Engineering, NMIMS University, Mumbai, India , January 2325, 2013.

[8] Arijit Kumar Pal, Poulami Das, Nilanjan Dey “Odd-Even Embedding Scheme Based Modified Reversible Watermarking Technique using Blueprint”, published in arXiv preprint arXiv: 1303.5972,2013.

[9] Nilanjan Dey, Prasenjit Maji , Poulami Das, Shouvik Biswas, Achintya Das, Sheli Sinha Chaudhuri, "Embedding of Blink Frequency in Electrooculography Signal using Difference Expansion based Reversible Watermarking Technique", Scientific Bulletin of the Politehnica University of Timisoara Transactions on Electronics and Communications p-ISSN 1583-3380, vol. 57(71), no. 2, 2012

[10] Nilanjan Dey, Shouvik Biswas, Poulami Das, Achintya Das, Sheli Sinha Chaudhuri," Lifting Wavelet Transformation Based Blind Watermarking Technique of Photoplethysmographic Signals in Wireless Telecardiology", Second World Congress on Information and Communication Technologies (WICT 2012), Trivandrum, India: October 30-November 02, 2012.

[11] Nilanjan Dey, Poulami Das, Achintya Das, Sheli Sinha Chaudhuri, "DWT-DCT-SVD Based Intravascular Ultrasound Video Watermarking "Second World Congress on Information and Communication Technologies (WICT 2012), Trivandrum, India: October 30-November 02, 2012.

[12] Baisa L. Gunjal, Suresh N. Mali,"ROI Based Embedded Watermarking of Medical Images for Secured Communication in Telemedicine" In International Journal of Computer and Communication Engineering. Vol. 6 (2012).

[13] Malay Kumar Kundu, Sudeb Das, "Lossless ROI Medical Image Watermarking Technique with Enhanced Security and High Payload Embedding" In International conference on Pattern Recognition. (1457-1460).

[14] Lin, C. P., Honye, J., Chang, C. J., \& Kuo, C. T.”Clinical Application of Intravascular Ultrasound in Coronary Artery Disease" An Update. ACTA Cardiol Sin. Received. (August 21, 2010).

[15] http://bme240.eng.uci.edu/students/06s/yuhsianh/ Quantitative\%20measurement.htm

[16] http://www.medicinenet.com/mri_scan/article.htm

[17] http://www.nlm.nih.gov/medlineplus/ency/article/003335.htm

[18] http://www.webmd.com/a-to-z-guides/magnetic-resonance-imaging-mri

[19] Jacob C Dunn," A Fuzzy Relative of the ISODATA Process and Its Use in Detecting Compact Well Separated Clusters" In J. Cyber. Vol. 3. pp. 32-57. (1973)

[20] James C. Bezdek, "Pattern Recognition with Fuzzy Objective Function Algorithms. In Plenum" New York, USA. (1981).

[21] Nilanjan Dey, Anamitra Bardhan Roy ,Moumita Pal, Achintya Das, "FCM Based Blood Vessel Segmentation Method For Retinal Images", International Journal of Computer Science and Network (IJCSN) (ISSN 2277- 5420), June 2012, Volume 1, Issue 3.

[22] Akhil pratap singh, Agya Mishra, "Wavelet Based Watermarking on Digital Image" In Indian Journal of Computer Science and Engineering. Vol. 1(2). pp. 86-91.

[23] Nilanjan Dey, Anamitra Bardhan Roy ,Sayantan Dey, "A Novel Approach of Color Image Hiding using RGB Color planes and DWT", International Journal of Computer Applications Volume 36- No.5, December 2011, (ISSN: 0975 - 8887),

[24] Chris Harris, Mike Stephens, "A Combined Corner and Edge Detector" In Proceedings of 4th Alvey Vision Conference. (1988).

[25] Konstantinos G. Derpanis, The Harris Corner Detector", 2004.

[26] http://ncalculators.com/statistics/signal-noise-ratio-calculation.htm. 